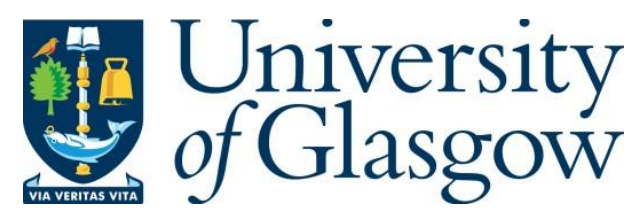

Kilgard, M. P., Rennaker, R. L., Alexander, J. and Dawson, J. (2018) Vagus nerve stimulation paired with tactile training improved sensory function in a chronic stroke patient. NeuroRehabilitation, 42(2), pp. 159-165.

There may be differences between this version and the published version. You are advised to consult the publisher's version if you wish to cite from it.

http://eprints.gla.ac.uk/159189/

Deposited on: 18 April 2018

Enlighten - Research publications by members of the University of Glasgow http://eprints.gla.ac.uk 


\title{
Vagus Nerve Stimulation Paired with Tactile Training Improved Sensory Function in a Chronic Stroke Patient
}

Michael P. Kilgard, PhD ${ }^{1 *}$, Robert L. Rennaker ${ }^{1,2}, \mathrm{PhD}$; Jen Alexander ${ }^{3}, \mathrm{MSc}$, Jesse Dawson, $\mathrm{MD}^{3}$

1. School of Behavioral and Brain Sciences, University of Texas at Dallas, Richardson, Texas, USA.

2. Department of Bioengineering, Erik Jonsson School of Engineering and Computer Science, University of Texas at Dallas, Richardson, Texas, USA.

3. Institute of Cardiovascular and Medical Sciences, College of Medical, Veterinary and Life Sciences, University of Glasgow, Western Infirmary, Glasgow, United Kingdom.

* Address for correspondence: Michael Kilgard, PhD, School of Behavioral and Brain Sciences, University of Texas at Dallas, BSB14, 800 West Campbell Road, Richardson, Texas, USA. Tel.: +1 972883 2339; Fax: +1 972883 2491; E-mail: kilgard@utdallas.edu.

\begin{abstract}
BACKGROUND: Recent studies indicate that vagus nerve stimulation (VNS) paired with rehabilitation can enhance neural plasticity in the primary sensory and motor cortices, improve forelimb function after stroke in animal models and improve motor function in patients with arm weakness after stroke.
\end{abstract}

OBJECTIVE: To gain "first-in-man" experience of VNS paired with tactile training in a patient with severe sensory impairment after stroke.

METHODS: During the long-term follow-up phase of a clinical trial of VNS paired with motor rehabilitation, a 71year-old man who had made good motor recovery had ongoing severe sensory loss in his left hand and arm. He received VNS paired with tactile therapy in an attempt to improve his sensory function. During twenty 2-hour sessions, each passive and active tactile event was paired with a 0.5 second burst of $0.8 \mathrm{~mA}$ VNS. Sensory function was measured before, halfway through, and after this therapy.

RESULTS: The patient did not report any side effects during or following VNS+Tactile therapy. Quantitative measures revealed lasting and clinically meaningful improvements in tactile threshold, proprioception, and stereognosis. After VNS+Tactile therapy, the patient was able to detect tactile stimulation to his affected hand that was eight times less intense, identify the joint position of his fingers in the affected hand three times more often, and identify everyday objects using his affected hand seven times more often, compared to baseline.

CONCLUSIONS: Sensory function significantly improved in this man following VNS paired with tactile stimulation. This approach merits further study in controlled clinical trials.

Keywords: Vagal Nerve Stimulation, neuromodulation, neuroplasticity, motor function, upper extremity 


\section{Introduction}

Impaired proprioception, stereognosis, and tactile sensation are common after stroke and are associated with poor functional outcomes (Kim, 1996). There is no established method to restore tactile sensation following stroke. However, recent evidence suggests that sensory retraining therapy can provide some benefit (Byl, 2003).

When delivered during physical therapy, vagus nerve stimulation (VNS) has shown promise in augmenting neuroplasticity and improving motor function after stroke (Dawson, 2016, 2016). VNS is used to reduce seizure frequency in some patients with epilepsy (Howland, 2014) with a 30 seconds on, 5 minutes off stimulation paradigm. Animal research indicates that brief, 0.5 second bursts of $0.8 \mathrm{~mA}$ VNS triggers release of norepinephrine and acetylcholine in the cerebral cortex, which can restore synaptic connectivity after brain injury and improve function if delivered during rehabilitative training (Engineer, 2011; Hays, 2014, 2014, Hulsey, 2016, 2017, Khodaparast, 2013, 2016; Nichols, 2011; Porter, 2012; Pruitt, 2016). It is not yet known, whether brief bursts of VNS paired with tactile discrimination training can improve the recovery of sensory function after stroke.

We report the use of VNS paired with tactile rehabilitation therapy in a patient with sensory impairments post stroke. The patient was included in a clinical study of VNS paired with physical therapy for upper extremity weakness (Dawson, 2016). The patient made good motor recovery but his function-limiting sensory impairments persisted.

\section{Methods}

2.1 Case description: The patient was a $87 \mathrm{~kg}$, 72-year-old right-handed male who suffered an embolic stroke at the age of 70. He had a right middle cerebral artery infarct visible on brain imaging. He consented to take part in a clinical trial of VNS paired with physical therapy for stroke. At study enrollment, he was eight months post stroke and had an Action Research Arm Test (ARAT) of 41. He consented to participate in an Institutional Review Board approved study of VNS delivered during stroke therapy (clinicaltrial.gov NCT01669161). The patient underwent VNS device implantation under general anesthetic (Fig. 1, Vivistim implantable pulse generator, MicroTransponder, Austin, TX). Additional details are available in an earlier report (Dawson, 2016). Following implantation, he had 6 weeks of motor therapy paired with VNS and his ARAT score improved to 48. Despite the motor gains from the VNS paired with physical therapy, the patient had residual, function-limiting sensory impairment. Thirty-three weeks after the end of physical therapy paired with VNS, and sixteen months after his stroke, the patient began tactile therapy paired with VNS. He had twenty sessions of VNS+Tactile therapy over five weeks.

\subsection{Sensory assessments:}

Detailed assessments of somatosensory function were completed before VNS+Tactile therapy, halfway through therapy, and after therapy. Tactile deficits were measured by the following examinations of the patient's left hand: von Frey filament detection threshold, identification of everday items, identification of changes in finger joint position, detection of light and deep touch, and two-point discrimination. Von Frey Filament testing was conducted at five different locations evenly distributed over the subject's left hand. Twenty different filament sizes (1.65-6.65) were tested at each site. Since the highest Von Frey Filament was $300 \mathrm{~g}$, the tactile threshold 
for each site was conservatively defined as the highest force tested that the subject could not detect.

Stereognosis was evaluated by allowing the blindfolded patient to active explore and attempt to name the following ten items: electrical plug, key, button, sponge, tennis ball, comb, toothbrush, wet face cloth, glass, and scissors. Two-point discrimination testing was conducted on the tip of all five fingers. Two-point discrimination testing began with a $15 \mathrm{~mm}$ separation. Separations were reduced by $1 \mathrm{~mm}$ at a time until a separation of 2 $\mathrm{mm}$ was reached or until the subject was unable to correctly identify the number of points (1 or 2) on seven out of ten tests. Each finger and the palm were tested for detection of light and deep touch. Detection of light touch was assessed using a cotton ball and detection of deep touch was assessed using a toothpick. Identification of joint position (extension or flexion) was evaluated five times on each finger.

\subsection{Functional Assessments:}

The following functional assessments were conducted before and after VNS + Tactile therapy: ARAT, Upper Extremity Fugl-Meyer (UEFM), Box and Block, Nine-Hole Peg Task (NHPT), grip strength and pinch strength.

\subsection{Intervention: VNS+Tactile therapy:}

Tactile therapy consisted of three daily exercises: passive localization, passive identification, and active object exploration. Each session was composed of six blocks of 40-50 trials. Each exercise was repeated during two blocks and the block order was shuffled across sessions. During passive localization the blindfolded patient's left hand was firmly contacted with an object (e.g. sand paper, toothpick, sponge, paint brush, cotton ball, pencil eraser) and the patient was asked to identify the location of contact (i.e. "tip" of named finger which was the palmar aspect of the distal phalanx, "base" of named finger which was the palmar aspect of the intermediate or proximal phalanges, or anywhere on the "palm"). Tactile stimuli were evenly distributed across 11 possible locations on the glabrous surface of the patient's left hand. During passive identification the patient was advised which objects would be used. He was then blindfolded and the subject's left hand was firmly contacted with an object and the blindfolded patient was asked to identify the object. Three different objects were used each session. The most frequently used were sandpaper, paintbrush, and toothpick, because these three were the most discriminable. During active exploration the patient was advised which objects would be used. The blindfolded patient then used his left hand to explore different objects and was asked to identify each object. Up to 7 different everyday objects were used each session, typically 5-7. The everyday objects included a water bottle, water bottle with ice in it, sponge, wet face cloth, toothpaste tube, toothbrush, electrical plug, plastic ball, comb, paper cup, tennis ball, tape roll, metal bolt with nut, pen, tape roll, hairbrush, and other items. The objects were placed in the subject's hand over a mat to reduce the potential to identify objects using auditory cues. Verbal feedback was provided for all three tasks. No quantitative data was collected during therapy sessions.

During each tactile stimulation event, a physical therapist pressed a button to trigger the stimulator to deliver a 0.5 second train of sixteen $0.8 \mathrm{~mA}$ pulses ( $30 \mathrm{~Hz}, 100$ us biphasic pulses) to the left vagus nerve. Each two-hour session resulted in $254 \pm 14$ (mean \pm STD) pairings of VNS with tactile stimulation. The median interval between VNS+Tactile pairing events was 16 seconds (interdecile range 9-53). Because of the brevity of the VNS bursts, the total VNS duration delivered in this study was 60 times less than in the standard VNS therapy for epilepsy (30 
seconds of VNS, followed by 5 minutes off). The total charge delivered was approximately 500 times less because the intensity and pulse width were lower than the standard VNS therapy for epilepsy.

\subsection{Statistical Analysis}

Statistical analysis was performed using the Kruskal-Wallis test. P-values $<0.05$ were considered statistically significant.

\section{Results}

Prior to the VNS+Tactile therapy, tactile stimulation of the patient's left hand was severely impaired (Fig. 2, Table 1). The patient was unable to detect the presence of Von Frey Filaments, except for the $300 \mathrm{~g}$ filament, which he was unable to detect three out of five times. The patient correctly identified only one of the ten items that he explored. The patient was only able to detect finger joint flexion or extension only four times out of twenty during the proprioception task. The patient was unable to detect any of the ten light and deep touch stimuli. The patient was unable to perform the two-point discrimination task even at the widest distance tested (15 mm). The patient's sensory impairment likely contributed to the low utility of his left hand in many activities of daily living. The patient performed within the normal range on all assessments when using his right hand.

The patient performed at chance during the first several therapy sessions. As sensory function improved over the course of therapy, the tasks were made more challenging such that performance during therapy was typically near $50 \%$ correct.

After ten days of VNS+Tactile Therapy, the patient demonstrated an $80 \%$ recovery in tactile threshold as measured with von Frey filaments (Fig. 2A, p<0.05). The other tactile measures improved, but not significantly after the first ten days of therapy. Upon completion of twenty days of VNS+Tactile therapy, the patient exhibited an $88 \%$ recovery of tactile threshold compared to pre-therapy threshold. Recovery of stereognosis, location, and detection were $70 \%, 60 \%$, and $50 \%$, respectively (Fig. 2B-D, $\mathrm{p}<0.05$ ). There was no detectable improvement in two-point discrimination.

VNS+Tactile therapy did not alter the patient's score on the ARAT. His UEFM score changed from 45 to 60 . His Box and Block score changed from 34 to 32 . His NHPT score changed from 77 to 110 seconds. His grip strength changed from 27 to $17 \mathrm{~kg}$. His pinch strength changed from 5.1 to $5.2 \mathrm{~kg}$.

\section{Discussion}

Sensory loss following stroke can severely limit activities of daily living and leisure activities. The patient in this study experienced both motor and sensory problems. Sensory function improved after tactile therapy was paired with VNS for several weeks. The patient self-reported that the additional tactile therapy with VNS was associated with functional improvements, such as an increased ability to use his walking stick in the affected hand and greater ease of household tasks.

Rehabilitative therapies are designed to promote adaptive circuit changes after neurological disease or injury, but insufficient neural plasticity often limits recovery. Somatosensory dysfunction is common in stroke patients and new therapies to enhance plasticity in sensory circuits are desperately needed to improve post- 
stroke sensation (Byl, 2003; Hays, 2013; Kim, 1996). The mechanisms responsible for the improvement seen in this patient are not yet known. Neural plasticity represents a plausible explanation, but it remains unknown if this patient's improvements were due to VNS, the tactile therapy itself or the combination of the two.

Behavioral deficits in animal models of chronic ischemic stroke, intracerebral hemorrhage, and traumatic brain injury improve significantly more when VNS is paired with rehabilitative training compared to rehabilitation alone (Hays, 2014; Khodaparast, 2013, 2014; Pruitt, 2016). Importantly, VNS provides no benefit when it is delivered two hours after the end of daily rehabilitation (Khodaparast, 2016). The receptive field plasticity that occurs when VNS is paired with sensory stimulation is specific to events that occur within a few seconds of VNS bursts (Engineer, 2011). These observations suggest that the combination of VNS and tactile stimulation was likely responsible for the gains observed in this patient. Though as yet unproven, we suspect that the enhanced sensory function in this patient resulted from extensive somatosensory receptive field plasticity, enhanced by the combination of VNS and tactile stimulation (Fig. 3) (Harrison, 2013; Hays, 2014; Jenkins, 1987; Khodaparast, 2013, 2014; Pruitt, 2016; Reinecke, 2003; Xerri, 1998).

Animal studies have consistently reported that stroke can cause somatosensory neurons in the cortex to lose responsiveness and selectivity (Harrison, 2013; Jenkins, 1987; Reinecke, 2003). The near complete lack of tactile sensation in this patient is consistent with these observations and suggests that few neurons were responsive to his left hand after stroke (Fig. 3, After Stroke). The modest improvement in tactile function during the first two weeks of VNS+Tactile therapy suggests that the therapy may have increased the number of neurons that were responsive to inputs from the left hand (Fig. 3, After 10 days of VNS+Tactile Therapy). Additional VNS+Tactile therapy may have improved somatosensory function in this patient by further increasing the number of somatosensory neurons that respond to the left hand and improving receptive field selectivity (Fig. 3, After 20 days of VNS+Tactile Therapy). The observation that VNS paired with tactile therapy improved sensory localization suggests that the therapy narrowed receptive fields from the entire hand to individual fingers, as in earlier animal studies (Xerri, 1998). Although VNS+Tactile pairing appears to have produced substantial recovery of sensory function in this chronic stroke patient, persistently elevated two point discrimination thresholds suggest that somatosensory receptive fields may remain abnormally large. It is possible that pairing VNS with more fine-grained tactile discrimination training would further narrow receptive fields and enhance sensory function. Future studies are needed to more closely characterize and optimize the effects of VNS+Tactile therapy on sensory receptive fields, and clarify the molecular mechanisms of action.

A number of studies indicate that VNS enhances neural plasticity by activating neuromodulatory systems in conjunction with training. VNS drives phasic activation the cholinergic basal forebrain and the noradrenergic locus coeruleus (Hulsey, 2017; Nichols, 2011). VNS must be delivered within a few seconds of the targeted movement to generate the maximum benefit (Hays, 2014; Khodaparast, 2016). Repeatedly pairing brief bursts of VNS with sensory or motor events drives robust, event-specific plasticity in neural circuits (Engineer, 2011; Porter, 2012). Lesion of the cholinergic neurons in the basal forebrain is sufficient to prevent the cortical motor map plasticity caused by VNS-movement pairing, highlighting the role of neuromodulatory systems in VNSdependent enhancement of plasticity (Hulsey, 2016). These studies suggest that it will be important to pay careful attention to patient medication and comorbid conditions that might interfere with neuromodulator function. 
Pairing VNS with sensory and motor experiences provides a novel method to enhance the neural plasticity that results from traditional rehabilitation. Although this report only represents a single anecdotal case, it emphasizes the potential of plasticity-based approaches to improve tactile function years after stroke and the need for further animal and human study.

\section{Acknowledgements}

The clinical trial was funded by MicroTransponder, Inc. Drs. Kilgard and Rennaker have received funding from the U.S. National Institutes of Health (R01NS085167 and R43NS084566) and Defense Advanced Research Projects Agency (DARPA) to support research involving vagus nerve stimulation. Their DARPA funding was supported by the Biological Technologies Office (BTO) Electrical Prescriptions (ElectRx) and Targeted Neuroplasticity Training (TNT) programs under the auspices of Dr. Doug Weber through the Space and Naval Warfare Systems Center, Pacific Cooperative Agreement Nos. HR0011-15-2-0017, N66001-15-2-4057, and N66001-17-2-4011 (RLR and MPK). The funders had no responsibility for the collection, analysis, and interpretation of study data. The funders had no responsibility for writing of the trial report, or in the decision to submit the article for publication. Drs. Nancy Byl, Jane Wigginton and Seth Hays provided valuable suggestions to improve the manuscript.

\section{Declaration of Interest}

Dr. Kilgard is a shareholder and consultant for MicroTransponder Inc. Dr. Dawson has received reimbursement for conference attendance where results of the study were presented from MicroTransponder Inc. Dr. Robert Rennaker and Jen Alexander state no conflicts of interest. 


\section{References}

Byl, N., Roderick, J., Mohamed, O., Hanny, M., Kotler, J., Smith, A., Tang, M., \& Abrams, G. (2003). Effectiveness of Sensory and Motor Rehabilitation of the Upper Limb Following the Principles of Neuroplasticity: Patients Stable Poststroke. Neurorehabilitation \& Neural Repair, 17, 176-191.

Dawson, J., \& McGrane, F. (2016). Vagus Nerve Stimulation and Upper Limb Rehabilitation. Current Physical Medicine and Rehabilitation Reports, 4, 186-189.

Dawson, J., Pierce, D., Dixit, A., Kimberley, T. J., Robertson, M., Tarver, B., Hilmi, O., Mclean, J., Forbes, K., Kilgard, M. P., Rennaker, R. L., Cramer, S. C., Walters, M., \& Engineer, N. (2016). Safety, feasibility, and efficacy of vagus nerve stimulation paired with upper-limb rehabilitation after ischemic stroke. Stroke, 47, 143-150.

Engineer, N. D., Riley, J. R., Seale, J. D., Vrana, W. A., Shetake, J. A., Sudanagunta, S. P., Borland, M. S., \& Kilgard, M. P. (2011). Reversing pathological neural activity using targeted plasticity. Nature, 470, 101-4.

Harrison, T. C., Silasi, G., Boyd, J. D., \& Murphy, T. H. (2013). Displacement of sensory maps and disorganization of motor cortex after targeted stroke in mice. Stroke, 44, 2300-2306.

Hays, S. A., Khodaparast, N., Hulsey, D. R., Ruiz, A., Sloan, A. M., Rennaker, R. L., \& Kilgard, M. P. (2014). Vagus nerve stimulation during rehabilitative training improves functional recovery after intracerebral hemorrhage. Stroke; a Journal of Cerebral Circulation, 45, 3097-3100.

Hays, S. A., Khodaparast, N., Ruiz, A., Sloan, A. M., Hulsey, D. R., Rennaker, R. L., \& Kilgard, M. P. (2014). The timing and amount of vagus nerve stimulation during rehabilitative training affect poststroke recovery of forelimb strength. NeuroReport, 25, 682-688.

Hays, S. A., Rennaker, R. L., \& Kilgard, M. P. (2013). Targeting plasticity with vagus nerve stimulation to treat neurological disease. Progress in Brain Research, 207, 275-299.

Howland, R. H. (2014). Vagus Nerve Stimulation. Current Behavioral Neuroscience Reports, 1, 64-73.

Hulsey, D. R., Hays, S. A., Khodaparast, N., Ruiz, A., Das, P., Rennaker, R. L., \& Kilgard, M. P. (2016). Reorganization of Motor Cortex by Vagus Nerve Stimulation Requires Cholinergic Innervation. Brain Stimulation, 9, 174-181.

Hulsey, D. R., Riley, J. R., Loerwald, K. W., Rennaker, R. L., Kilgard, M. P., \& Hays, S. A. (2017). Parametric characterization of neural activity in the locus coeruleus in response to vagus nerve stimulation. Experimental Neurology, 289, 21-30.

Jenkins, W. M., \& Merzenich, M. M. (1987). Chapter 21 Reorganization of neocortical representations after brain injury: a neurophysiological model of the bases of recovery from stroke. Progress in Brain Research, 71, 249-266.

Khodaparast, N., Hays, S. A., Sloan, A. M., Fayyaz, T., Hulsey, D. R., Rennaker, R. L., \& Kilgard, M. P. (2014). Vagus Nerve Stimulation Delivered During Motor Rehabilitation Improves Recovery in a Rat Model of Stroke. Neurorehabilitation and Neural Repair, 28, 698-706.

Khodaparast, N., Hays, S. A., Sloan, A. M., Hulsey, D. R., Ruiz, A., Pantoja, M., Rennaker, R. L., \& Kilgard, M. P. (2013). Vagus nerve stimulation during rehabilitative training improves forelimb strength following ischemic stroke. Neurobiology of Disease, 60. doi:10.1016/j.nbd.2013.08.002

Khodaparast, N., Kilgard, M. P., Casavant, R., Ruiz, A., Qureshi, I., Ganzer, P. D., Rennaker, R. L., \& Hays, S. A. (2016). Vagus Nerve Stimulation During Rehabilitative Training Improves Forelimb Recovery After Chronic Ischemic Stroke in Rats. Neurorehabilitation and Neural Repair, 30, 676-684.

Kim, J. S., \& Choi-Kwon, S. (1996). Discriminative sensory dysfunction after unilateral stroke. Stroke; a Journal of Cerebral Circulation, 27, 677-82.

Nichols, J. A., Nichols, A. R., Smirnakis, S. M., Engineer, N. D., Kilgard, M. P., \& Atzori, M. (2011). Vagus nerve stimulation modulates cortical synchrony and excitability through the activation of muscarinic receptors. Neuroscience, 189, 207-214.

Porter, B. A., Khodaparast, N., Fayyaz, T., Cheung, R. J., Ahmed, S. S., Vrana, W. A., Rennaker, R. L., \& Kilgard, M. 
P. (2012). Repeatedly pairing vagus nerve stimulation with a movement reorganizes primary motor cortex. Cerebral Cortex, 22, 2365-2374.

Pruitt, D. T., Schmid, A. N., Kim, L. J., Abe, C. M., Trieu, J. L., Choua, C., Hays, S. A., Kilgard, M. P., \& Rennaker, R. L. (2016). Vagus Nerve Stimulation Delivered with Motor Training Enhances Recovery of Function after Traumatic Brain Injury. Journal of Neurotrauma, 33, 871-879.

Reinecke, S., Dinse, H. R., Reinke, H., \& Witte, O. W. (2003). Induction of bilateral plasticity in sensory cortical maps by small unilateral cortical infarcts in rats. European Journal of Neuroscience, 17, 623-627.

Xerri, C., Merzenich, M. M., Peterson, B. E., \& Jenkins, W. (1998). Plasticity of primary somatosensory cortex paralleling sensorimotor skill recovery from stroke in adult monkeys. Journal of Neurophysiology, 79, 21192148. 


\section{Tables}

Table 1.

\begin{tabular}{rlll}
\hline Left Hand Sensory Assessments & $\begin{array}{c}\text { Before } \\
\text { Therapy }\end{array}$ & $\begin{array}{c}\text { Mid- } \\
\text { Therapy }\end{array}$ & $\begin{array}{c}\text { After } \\
\text { Therapy }\end{array}$ \\
\hline Von Frey Filament Detection Threshold & $212 \pm 86 \mathrm{~g}$ & $43 \pm 27 \mathrm{~g} *$ & $26 \pm 17 \mathrm{~g} *$ \\
Correct Identification of Everyday Objects & $1 / 10$ & $4 / 10$ & $7 / 10^{*}$ \\
Correct Identification of Joint Position & $4 / 25$ & $6 / 25$ & $15 / 25^{*}$ \\
Detection of Light Touch & $0 / 6$ & $2 / 6$ & $6 / 6 *$ \\
Detection of Deep Touch & $0 / 6$ & $3 / 6$ & $3 / 6$ \\
Two Point Discrimination & $>15 \mathrm{~mm}$ & $>15 \mathrm{~mm}$ & $>15 \mathrm{~mm}$ \\
\hline
\end{tabular}

$(* \mathrm{P}<0.05)$ 
Figures

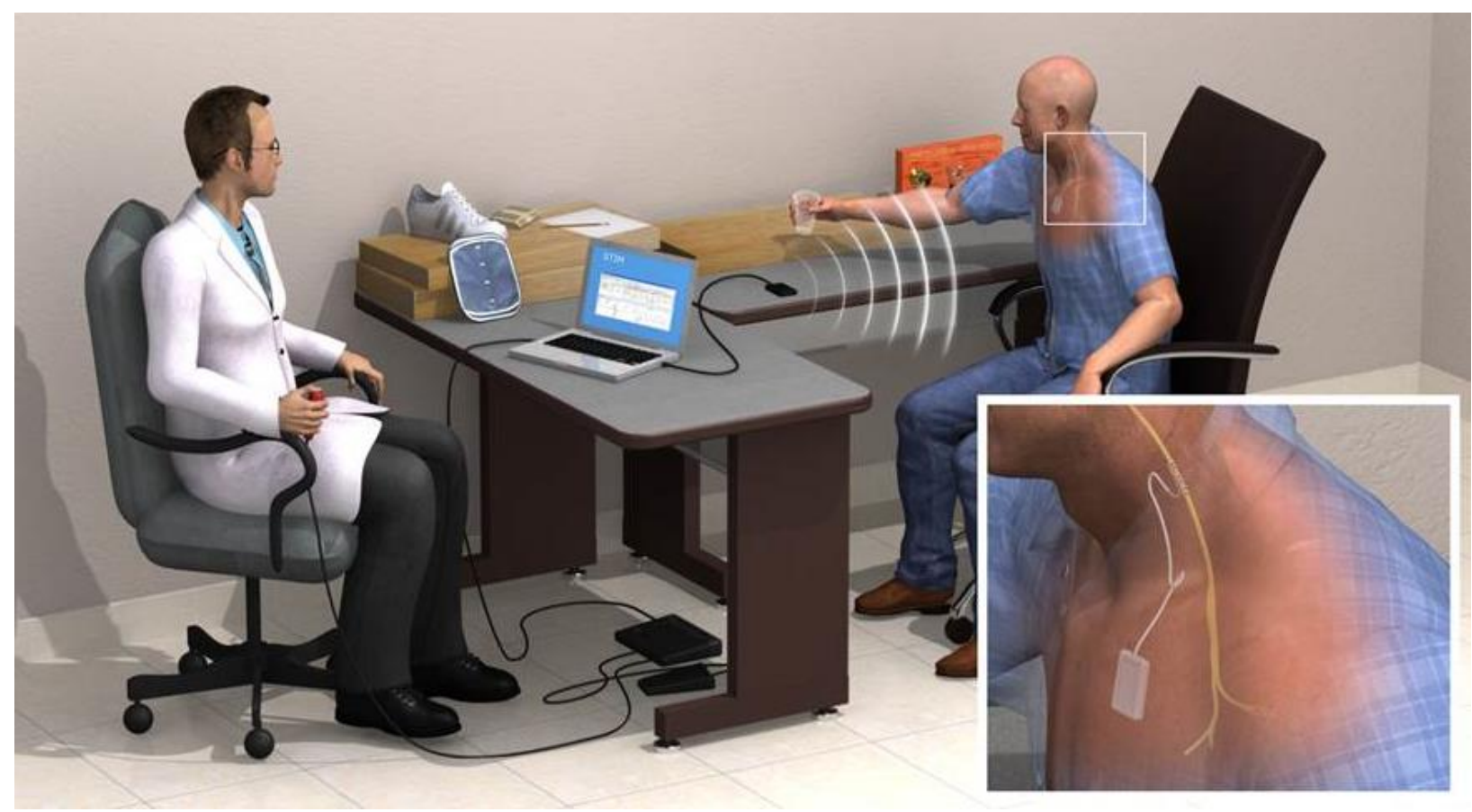

Figure 1. Implanted device allows brief bursts of vagus nerve stimulation to be delivered at precisely at the moment of individual motor training events (in a previous study) or during tactile sensory stimulation events (in the current study). 

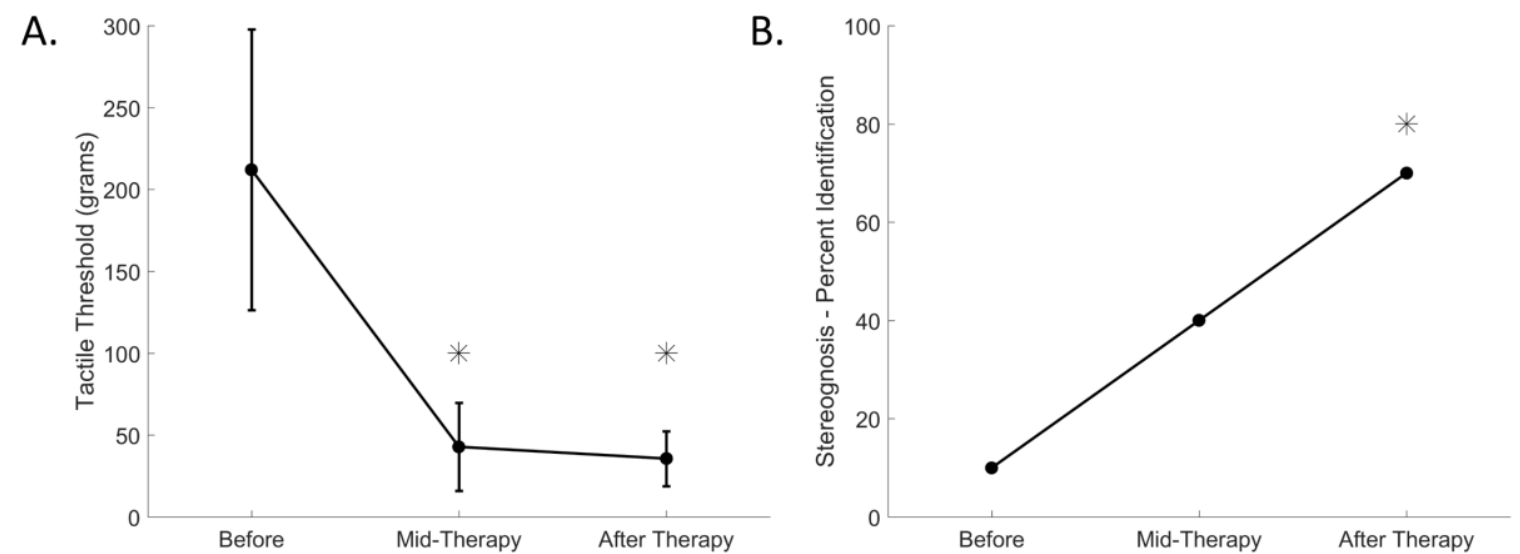

C.

D.
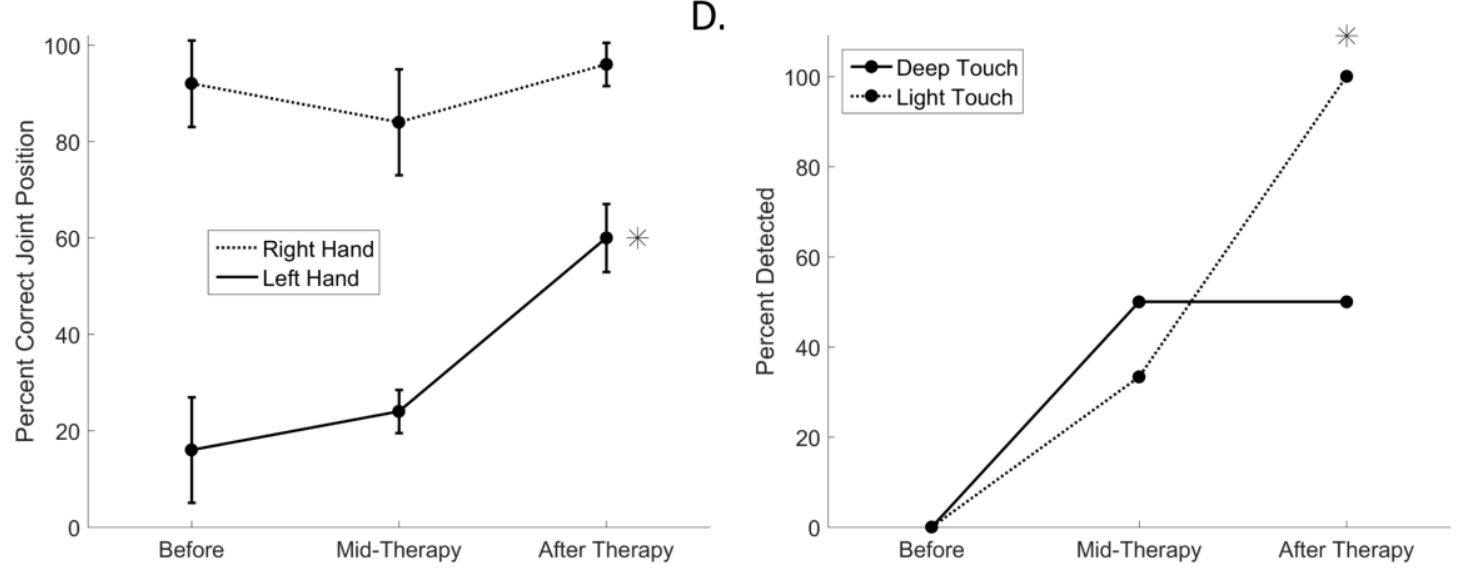

Figure 2. Vagus nerve stimulation paired with tactile stimulation significantly improved sensory function. Error bars indicate standard error of the mean. 


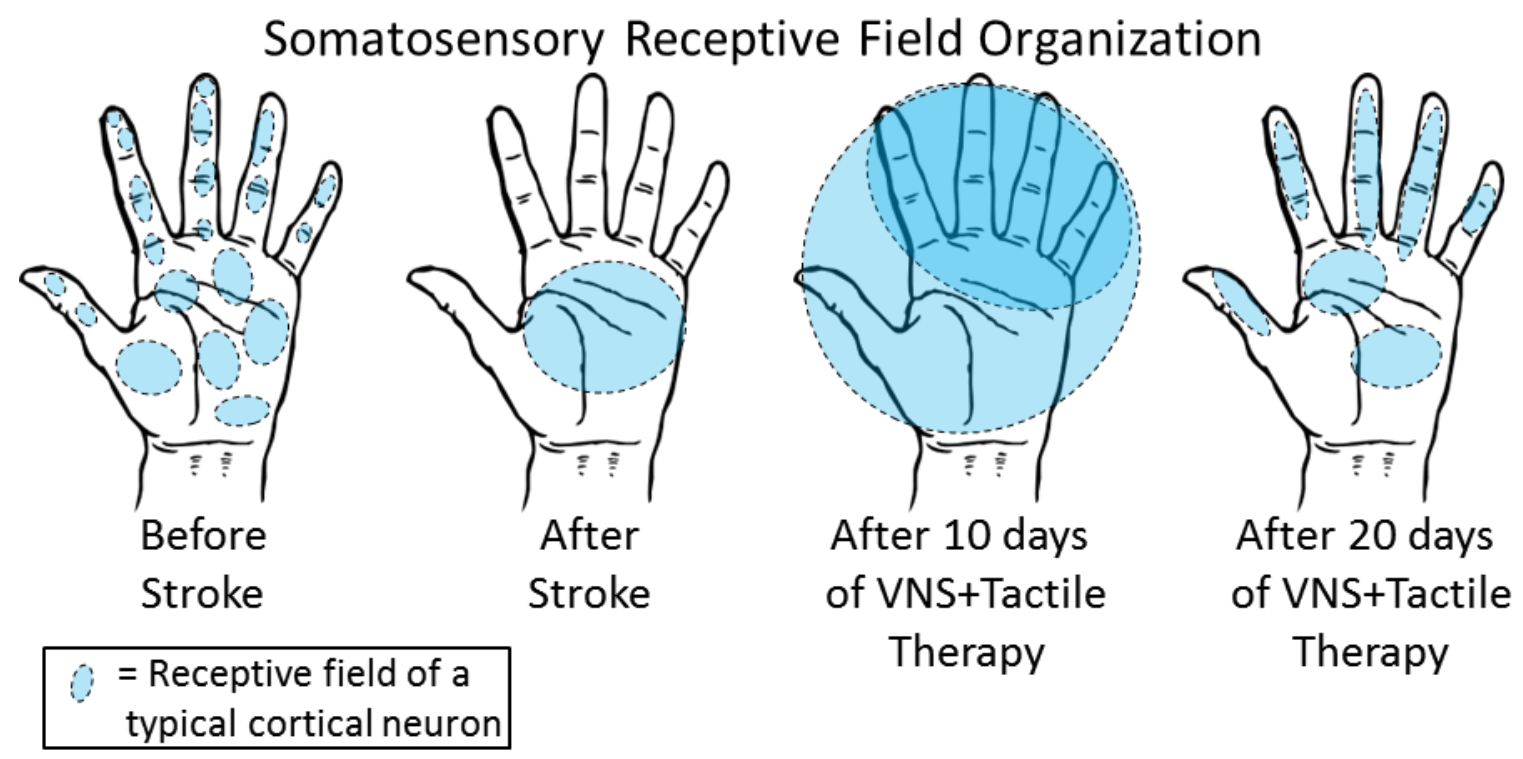

Figure 3. Schematic Illustration of the possible changes in somatosensory receptive field structure following stroke and after tactile therapy paired with VNS. Sensory function in this patient was very poor more than a year after his stroke. Many fewer cortical neurons likely provided useful tactile information compared to pre-stroke. The progressive sensory improvement during twenty days of VNS+Tactile therapy could be explained by a gradual recovery of somatosensory receptive fields. Both the number of responsive neurons and their spatial selectivity may have increased. The patient's persistent inability to perform two-point discrimination at $15 \mathrm{~mm}$ could be explained by receptive fields that remained substantially larger than before stroke. 
